( С. С. Мустафина, О.Д. Рымар, С.К. Малютина, Д.В. Денисова, Л.В. Щербакова, М.И. Воевода

ФГБНУ ФИЦ Институт цитологии и генетики СО РАН, Новосибирск

ЦЕль. Оценить распространенность сахарного диабета 2 типа (СД2) в разных возрастных группах взрослого населения Новосибирска по данным эпидемиологических исследований 2003-2005 и 2013-2016 гг.

МЕтоды. В период 2003-2005 гг. проведено одномоментное обследование репрезентативной популяционной выборки мужчин и женщин 45-69 лет - жителей двух административных районов г. Новосибирска в рамках международного проеKTa HAPIEE.

В 2013-2016 гг. проводилось популяционное обследование случайной репрезентативной выборки населения 25-44 лет обоего пола - жителей одного из районов Новосибирска.

Для постановки диагноза СД2 использованы критерии АДА (2003, 2013 гг.): уровень глюкозы плазмы натощак $\geq 7,0$ ммоль/л однократно, после 8-часового голодания. Также в группу с СД2 вошли лица с уровнем глюкозы натощак <7,0 ммоль/л, но указавшие, что имеют СД2 и получают соответствующее лечение. Для выявления нарушенной гликемии натощак (НГН) использованы рекомендации ВО3, 1999 г., согласно которым НГН определяется при уровне гликемии натощак от 6,1 до 6,9 ммоль/л. Нормогликемия оценивалась как показатель глюкозы плазмы менее 6,1ммоль/л.

РЕЗУЛЬТАТЫ. Среди жителей 45-69 лет, по данным популяционного скрининга 2003-2005 гг., распространенность СД2 составила 11,3\%. Распространенность СД2 по результатам скрининга 2013-2016 гг. среди жителей 25-44 лет - 2,2\%, выше

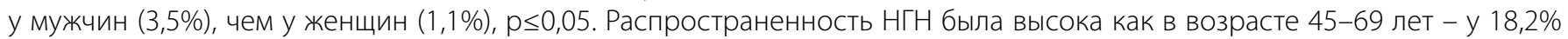
(2003-2005 гг.), так и в молодой возрастной группе 25-44 лет - 21,6\% (2013-2016 гг.), что особенно вызывает тревогу.

Менее половины (4,7 из 11,3\%) обследованных в возрасте 45-69 лет и только 1 человек в возрасте 25-44 лет 3нали, что у них есть СД2, что свидетельствует о недостаточном уровне знаний сибиряков о проблеме Сд2.

ЗАКЛЮЧЕНИЕ. Среди взрослого населения 45-69 лет в 2003-2005 гг. у 18,2\% наблюдалась НГН, у 11,3\% - СД2. У лиц 25-44 лет в 2013-2016 гг. выявлена НГН у 21,6\%, СД2 - у 2,2\%.

кЛючеВыЕ СлОВА: сахарный диабет 2 типа; эпидемиологическое исследование; недиагностированный сахарный диабет; распространенность; Новосибирск

\title{
PREVALENCE OF DIABETES IN THE ADULT POPULATION OF NOVOSIBIRSK
}

(c) Svetlana V. Mustafina, Oksana D. Rymar, Sofiya K. Malyutina, Diana V. Denisova, Liliya V. Shcherbakova, Mihail I. Voevoda

Institute of Cytology and Genetics, Novosibirsk, Russia

AIMS. To estimate the prevalence of type 2 diabetes mellitus (T2DM) in different age groups of the adult population of Novosibirsk, according to epidemiological studies in 2003-2005 and 2013-2016.

METHODS. We examined a representative population sample (assessed in 2003-2005) of men and women aged 45-69 years in two administrative districts of Novosibirsk, as a part of the international HAPIEE project. According to the tables of random numbers, representative samples of men and women aged 45-69 years were formed, to which letters were sent, inviting them to pass for examination. During 2013-2016, a second population survey was conducted on a random representative sample of a population of 25-44-year-olds of both sexes. Participants were residents of one of the districts of Novosibirsk. T2DM and impaired fasting glucose (IFG) were diagnosed using fasting plasma glucose (FPG) levels (diabetes: FPG $\geq 7.0$ mmol/l; IFG: FPG 6.1-6.9 mmol/I).

RESULTS. The prevalence of T2DM among residents aged 45-69 years was 11.3\%, and overall, no significant difference in prevalence was found between females and males (11.3\% vs. 11.0\%).

However, the overall prevalence of T2DM among residents aged 25-44 years was 2.2\%, and prevalence was higher in men (3.5\%) than in women (1.1\%), $\mathrm{p} \leq 0.05$.

High prevalence of IFG was found in the 45-69 age group (18.2\%, in 2003-2005), and in the younger age group of 25-44 years (21.6\%, in 2013-2016). The high rate among young individuals is particularly alarming. 
Less than one half (4.8 out of 11.3\%) of participants aged 45-69 tested positive for T2DM, and only one in the age group 25-44 years knew he/she had diabetes. This indicates a lack of knowledge among Siberians about their problem with diabetes.

CONCLUSION. Approximately one in five adults had IFG. Among the adult population aged 45-69 (in 2003-2005), 18.2\% had IFG and 11.3\% had T2DM. In individuals aged 25-44 years in 2013-2016, IFG was observed in 21.6\%, and 2.2\% had T2DM ( $p \leq 0.05$ ).

KEYWORDS: diabetes mellitus; epidemiological study; undiagnosed diabetes mellitus; prevalence; Novosibirsk

Распространенность сахарного диабета 2 типа (СД2) в Сибирском федеральном округе (СФО) изучена недостаточно. СФО является высокоразвитым индустриальным и культурным округом с мощным научным потенциалом. В регионе производится более 20\% промышленной продукции страны. В состав СФО входят 12 субъектов Российской Федерации: Республика Алтай, Республика Бурятия, Республика Тыва, Республика Хакасия, Алтайский край, Красноярский край, Забайкальский край, Иркутская, Кемеровская, Новосибирская, Омская, Томская области. Площадь территории СФО составляет 5145,0 тыс. км² (29\% площади территории России). Численность населения СФО на 2016 г. составляла 19324031 человек, из них на долю городского населения приходилось 70,7\%, сельского - 29,3\%. Крупнейшими городами СФО являются Новосибирск, Омск, Красноярск, Иркутск, Барнаул, Новокузнецк, Кемерово, Томск, Улан-Удэ, Чита. Всего на территории округа расположены 132 города. По плотности населения СФО занимает предпоследнее место среди федеральных округов: всего 3,8 чел. на км². При этом население СФО размещено по его территории неравномерно. В Кемеровской области плотность населения составляет 31,6 чел. на км², в то время как на севере Красноярского края плотность населения составляет 0,3-0,5 чел. на км². Центр округа - г. Новосибирск (в 2016 г. в Новосибирске проживали 1584138 человек) [1].

Институт терапии с первых лет своего существования занимается изучением сахарного диабета (СД) и других неинфекционных заболеваний в разных регионах Сибири, используя для этого различные методы [2, 3, 4]. В первую очередь, проводится анализ данных официальной статистики, основанной на учете обращаемости заболевших. С 1985 г. НИИ терапии учрежден регистр СД в одном из административных районов Новосибирска. Метод регистров позволяет полнее учитывать количество заболевших СД, анализируя при этом ряд дополнительных параметров. В конце 1990-х гг. главному эндокринологу Минздравсоцразвития России академику И.И. Дедову и команде сотрудников удалось внедрить в масштабе всей страны Государственную систему регистра СД (постановление Правительства РФ от 07.10.96 № 1171 «О федеральной целевой программе "Сахарный диабет"»), что стало, несомненно, большим достижением. Новосибирский городской регистр СД введен в действие в 1999 г. и с 2002 г. является частью Государственного регистра СД [5]. Для более полного суждения о фактической частоте (распространенности) среди населения СД используются методы эпидемиологических исследований, в частности скрининги репрезентативных групп населения.

Более глубокие исследования по оценке распространенности СД в популяции выполнены в Новосибирске в 2003-2005 гг. Этот фрагмент исследований был включен в качестве факультативного в большой международный проект HAPIEE (Детерминанты сердечно-сосудистых забо- леваний в Восточной Европе: многоцентровое когортное исследование) [4]. В последующие годы и по настоящее время проводится наблюдение за изучаемой когортой. Наряду с этим, с 2013 г. по настоящее время проводится популяционное обследование случайной репрезентативной выборки населения 25-44 лет обоего пола - жителей одного из типичных районов Новосибирска.

\section{ЦЕЛЬ}

Оценить распространенность СД2 в разных возрастных группах взрослого населения Новосибирска по данным эпидемиологических исследований 2003-2005 гг. и 2013-2016 гг.

\section{МЕТОДЫ}

Дизайн исследования, критерии соответствия, условия проведения, продолжительность исследования

Город Новосибирск является типичным для Сибири крупным индустриальным центром. Общая численность жителей 2 районов всех возрастов составляет 346 тыс. человек, в возрасте 45-69 лет - 96 тыс. человек. В период 2003-2005 гг. проведено обследование репрезентативной популяционной выборки мужчин и женщин 45-69 лет двух типичных административных районов г. Новосибирска в рамках международного проекта HAPIEE (главные исследователи д.М.Н., проф. Малютина С.К., акад. РАН Никитин Ю.П.). По таблицам случайных чисел на основе избирательных списков были сформированы репрезентативные выборки мужчин и женщин в возрасте 45-69 лет, которым были разосланы письма, приглашающие их пройти обследование. Отклик (процент пришедших на обследование) составил 70\%. Было обследовано 9360 человек [4].

В течение 2013-2016 гг. проводилось популяционное обследование случайной репрезентативной выборки населения 25-44 лет обоего пола - жителей одного из типичных районов Новосибирска. Обследованы 1331 человек.

\section{Описание медицинского вмешательства}

Протоколы исследования включали: анкетирование, оценку социально-демографических, антропометрических данных, определение биохимических показателей.

Для определения глюкозы забор крови проводился из локтевой вены вакутейнером, в положении сидя, натощак, после 10-часового голодания. После центрифугирования сыворотку хранили в низкотемпературной камере $\left(-70^{\circ} \mathrm{C}\right)$. Определение содержания уровня гликемии проводилось энзиматическими методами с использованием стандартных реактивов BIOKON на биохимическом анализаторе FP-901 LabSystem. Пересчет глюкозы сыворотки крови в глюкозу плазмы осуществлялся по формуле: глюкоза плазмы $($ ммоль/л) $=-0,137+1,047 \times$ глюкоза сыворотки 
(ммоль/л) (EASD, 2005 г.). Для постановки диагноза СД2 использованы критерии Американской диабетической ассоциации (АДА, 2003, 2013 гг.): уровень глюкозы крови натощак $\geq 7,0$ ммоль/л однократно, после 8-часового голодания [7]. Также в группу с СД2 вошли лица с уровнем глюкозы крови натощак <7,0 ммоль/л на момент исследования, но указавшие, что имеют СД2 и, по данным анамнеза, получающие лечение. Для выявления нарушенной гликемии натощак $(\mathrm{HГH})$ были использованы рекомендации Всемирной Организации Здравоохранения (ВО3), принятые в 1999 г., согласно которым НГН определяется при уровне гликемии натощак от 6,1 до 6,9 ммоль/л, и критерии АДА, 2003 г., 2013 г., по которым НГН определяется при уровне гликемии натощак от 5,6 до 6,9 ммоль/л [7, 8, 9]. Нормогликемия оценивалась как показатель глюкозы плазмы крови менее 6,1 ммоль/л [7].

\section{Этическая экспертиза}

Данный протокол соответствует нормам GCP, одобрен в локальном этическом комитете «НИИТПМ» (Протокол №1 от 14.03.2002). Все участники исследования подписывали информированное согласие.

\section{Статистический анализ}

Статистическая обработка полученных результатов проведена с помощью пакета SPSS V-13 и включала создание базы данных, автоматизированную проверку качества подготовки информации и статистический анализ. Статистическую значимость различий оценивали по критерию Стьюдента (t) при наличии двух групп; если анализировалось более двух групп, использовали однофакторный дисперсионный анализ; для сравнения качественных признаков применялся метод Пирсона $\left(X^{2}\right)$. Полученные данные в таблицах и тексте представлены как абсолютные и относительные величины (n, \%). Различия рассматривали как статистически значимые при $p<0,05, p \leq 0,01$ - очень значимые, $p \leq 0,001$ - высокозначимые.

\section{РЕЗУЛЬТАТЫ}

\section{Объекты (участники) исследования}

Из 9360 человек 45-69 лет, обследованных на этапе скрининга, не было результатов глюкозы плазмы у 205 человек, поэтому они не были включены в анализ. Изучена распространенность СД2 в репрезентативной выборке из 9155 человек обоего пола жителей Новосибирска: 4169 (45,5\%) мужчин и 4986 (54,5\%) женщин.

Таблица 1. Распространенность СД2 у мужчин и женщин 45-69 лет в зависимости от возраста обследованных

\begin{tabular}{ccccc}
\hline $\begin{array}{c}\text { Возраст, } \\
\text { лет }\end{array}$ & $\begin{array}{c}\text { Мужчины, } \\
\%\end{array}$ & $\begin{array}{c}\text { Женщины, } \\
\%\end{array}$ & $\begin{array}{c}\text { Оба } \\
\text { пола, } \%\end{array}$ & $\begin{array}{c}\mathrm{P} \\
\text { м/ж }\end{array}$ \\
\hline $45-49$ & 7,4 & 5,2 & 6,2 & 0,24 \\
$50-54$ & 10,0 & 8,3 & 9,1 & 0,08 \\
$55-59$ & $11,3^{*}$ & $11,5^{* *}$ & $11,4^{* *}$ & 0,85 \\
$60-64$ & $11,7^{*}$ & $15,0^{* *}$ & $13,5^{* *}$ & 0,07 \\
$65-69$ & $13,4^{* *}$ & $16,4^{* *}$ & $15,0^{* *}$ & 0,13 \\
$45-69$ & 11,0 & 11,6 & 11,3 & 0,35 \\
\hline
\end{tabular}

Примечания: $\mathrm{p}^{*}<0,05, \mathrm{p}^{* *}<0,001$ статистическая значимость различий возрастных диапазонов по сравнению с возрастом 45-49 лет; р м/ж достоверность различий между мужчинами и женщинами.
Основные результаты исследования

С учетом вышеперечисленных критериев (см. Материалы и методы) распространенность СД2 по данным популяционного скрининга 2003-2005 гг. среди жителей 45-69 лет в г. Новосибирске составила 11,3\% (1036 человек), среди мужчин - 11,0\% (459 человека), среди женщин - 11,6\% (577 человек), р>0,05. В табл. 1 представлена частота СД2 в изучаемых подгруппах. С увеличением возраста отмечается достоверный рост распространенности СД2 (табл. 1).

У 10,0\% (911 человек) определено повышение глюкозы крови выше 7,0 ммоль/л, у 1,3\% (125 человек) ранее был выставлен диагноз СД2 и они имели показатели гликемии натощак менее 7,0 ммоль/л. Из 10,0\% (911 человек) с повышенным уровнем глюкозы крови выше 7,0 ммоль/л знали, что у них СД2, 3,4\% (313 человек). Таким образом, из 11,3\% лиц с СД2 знали о своем заболевании 4,7\%. Во всей обследованной выборке определены значения глюкозы выше $\geq 11,1$ ммоль/л у 2,4\% (220 человек).

Проведен анализ терапии у 315 человек, указавших, что принимают сахароснижающие препараты. 261 пациент (82,9\%) принимали пероральные сахароснижающие препараты и соблюдали рекомендации по питанию; средний уровень глюкозы крови в этой группе составил 10,5 $\pm 3,7$ ммоль/л; 45 человек (14,3\%) получали инсулинотерапию и соблюдали рекомендации по питанию, средний уровень глюкозы крови в этой группе составил 11,1 1,7 ммоль/л; 9 человек (2,8\%) указали, что принимали пероральные сахароснижающие препараты, получали инсулинотерапию и соблюдали рекомендации по питанию, средний уровень глюкозы крови в этой группе составил

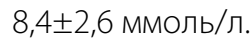

Среди лиц 45-69 лет с ранее не диагностированным СД2 во время скрининга среднее значение глюкозы плазмы натощак составило 8,8 2,8 ммоль/л, что значимо ниже, чем у пациентов с ранее диагностированным СД2 и получавших лечение - 10,5 33,9 ммоль/л, $\mathrm{p}=0,015$.

Среди участников 45-69 лет в Новосибирске НГН по критерию ВО3, 1999 г. (от 6,1 до 6,9 ммоль/л), выявлена у 18,2\% (1667 человек); по критерию АДА, 2003 г., 2013 г. (от 5,6 до 6,9 ммоль/л), - у 44,2\% (4051 человек).

Во время проведения скрининга обследуемым было предложено ответить на вопрос: «Говорили ли вам, что у вас СД?». Был предложен ответ: 1 - да, говорили и 2 нет, не говорили. При опросе выявлено, что знали о наличии у них СД 43,0\% опрошенных с СД2. Лучше о своем заболевании информированы женщины (табл. 2). С возрастом информированность о наличии СД2 увеличивалась

Таблица 2. Информированность о СД2 в зависимости от возраста обследованных

\begin{tabular}{lccc}
\hline Возраст, лет & Мужчины, \% & Женщины, \% & $\begin{array}{c}\text { Оба пола, } \\
\%\end{array}$ \\
\hline $45-49$ & 18,4 & 45,6 & 31,6 \\
$50-54$ & 31,3 & 43,8 & 37,4 \\
$55-59$ & 26,5 & 49,6 & 39,2 \\
$60-64$ & $38,7^{*}$ & $49,6^{*}$ & $45,3^{*}$ \\
$65-69$ & $41,2^{* *}$ & $54,7^{* *}$ & $49,7^{* *}$ \\
$45-69$ & 33,3 & 50,2 & 42,7 \\
\hline
\end{tabular}

Примечания: $\mathrm{p}^{*}<0,05, \mathrm{p}^{* *}<0,001$ - статистическая значимость различий возрастных диапазонов по сравнению с возрастом 45-49 лет. 
Таблица 3. Возрастная характеристика распространенности СД2 у мужчин и женщин 25-44 лет

\begin{tabular}{ccccc}
\hline $\begin{array}{c}\text { Возраст, } \\
\text { лет }\end{array}$ & $\begin{array}{c}\text { Мужчины, } \\
\%\end{array}$ & $\begin{array}{c}\text { Женщины, } \\
\%\end{array}$ & $\begin{array}{c}\text { Оба } \\
\text { пола, \% }\end{array}$ & $\begin{array}{c}\mathrm{P} \\
\text { м/ж }\end{array}$ \\
\hline $25-29$ & - & - & - & \\
$30-34$ & 2,4 & 0,7 & 1,6 & 0,208 \\
$35-39$ & 2,1 & 1,8 & 2,0 & 0,853 \\
$40-44$ & $7,7^{*}$ & 1,6 & $4,5^{*}$ & 0,006 \\
$25-44$ & 3,5 & 1,1 & 2,2 & 0,006 \\
\hline
\end{tabular}

Примечания: $\mathrm{p}^{*}=0,03$ - статистическая значимость различий возрастных диапазонов по сравнению с возрастом 30-34 лет

как у мужчин, так и у женщин.

При проверке данных обследования возрастной группы 25-44 лет из 1331 человека, обследованных на этапе скрининга, не было результатов глюкозы плазмы у 125 человек, поэтому они не были включены в анализ. Определена глюкоза плазмы крови у 1206 человек, 579 (48\%) мужчин и 627 (52\%) женщин. Распространенность СД2 по данным популяционного скрининга 2013-2015 гг. среди жителей 25-44 лет в г. Новосибирске составила 2,2\% (27 человек), выше среди мужчин - 3,5\% (20 человек), чем среди женщин - 1,1\% (7 человек), p $\leq 0,05$. В табл. 2 представлена частота СД2 в изучаемых подгруппах. Из 27 человек, у которых определен уровень глюкозы плазмы натощак $\geq 7$ ммоль/л, знал о наличии СД2 только 1 человек.

В молодой возрастной группе (25-44 лет) обращает внимание высокая частота НГН по критерию ВО3, 1999 г. (от 6,1 до 6,9 ммоль/л) - 21,6\% (261 человек); по критерию АДА, 2003 г., 2013 г. (от 5,6 до 6,9 ммоль/л) - 56,7\% (684 человека) (табл. 3).

\section{ОБСУЖДЕНИЕ}

\section{Резюме основного результата исследования,}

обсуждение основного результата исследования

Получены высокие показатели (без значимых гендерных различий) распространенности СД2 в популяции жителей Новосибирска 45-69 лет - 11,3\% в период 2003-2005 гг. Представленные нами данные в изучаемых возрастных подгруппах сопоставимы с данными исследования NATION, которое проводилось в России в 2013-2015 гг., несмотря на использование разных критериев для постановки диагноза СД2 (рис. 1) [10]. В исследовании в Новосибирске

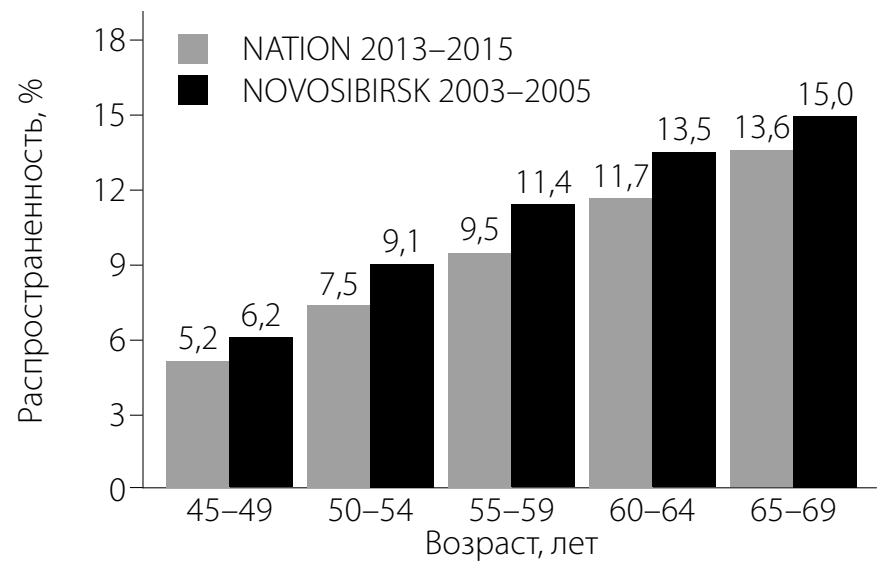

Рис. 1. Распространенность СД2 у лиц 45-69 лет. использовался показатель глюкозы плазмы натощак, в исследовании NATION - гликированный гемоглобин ( $\left.\mathrm{HbA}_{1 с}\right)$. Известно, что использование для диагностики СД критерия $\mathrm{HbA}_{1 с}$ дает более низкие показатели распространенности СД, чем с использованием глюкозы плазмы натощак или 2-часового перорального глюкозотолерантного теста [9]. Не представляется возможным сопоставить показатели распространенности СД2 у лиц 45-69 лет, полученные в результате популяционного скрининга в Новосибирске в период 2003-2005 гг., с более ранними популяционными исследованиями в России, так как в них не анализировались отдельно изучаемые возрастные подгруппы [11, 19].

С увеличением возраста отмечается достоверный рост распространенности СД2 у жителей Новосибирска, 6,2\% в возрасте 45-49 лет и с максимальной частотой в возрастной группе 65-69 лет - 15,0\%. По данным исследования NATION, также наиболее высокая частота СД2 отмечалась у людей в возрасте 65-69 лет - 13,6\%. Как справедливо отмечают авторы исследования NATION, полученные сведения важны, так как в этой возрастной группе наблюдается высокая распространенность заболеваний сердечно-сосудистой системы, терапия которых становится более сложной при наличии сопутствующего СД [13-16].

В возрастной группе 45-54 лет СД2 выявлялся чаще у мужчин, чем у женщин, после 60 лет - у женщин чаще, чем у мужчин, однако разница не достигла статистической значимости. В исследовании NATION скорректированная по возрасту распространенность СД2 также не выявила статистически значимого отличия. Помимо этого, авторы исследования отмечают, что при рассмотрении отдельных возрастных групп в исследовании NATION более высокая распространенность СД2 у женщин по сравнению с мужчинами была статистически значима только у лиц $\geq 60$ лет, что может быть обусловлено систематической ошибкой, связанной с выживаемостью анализируемых групп, если принять во внимание, что риск смерти от сердечно-сосудистой патологии выше у мужчин, чем у женщин [16-20].

Обращает на себя внимание то, что среди пациентов 45-69 лет с ранее не диагностированным СД2 и у тех, у которых обнаружены значения глюкозы крови более 7,0 моль/л во время скрининга среднее значение глюкозы плазмы натощак составило 8,8 22,8 ммоль/л, что было значимо ниже, чем у пациентов с ранее диагностированным СД2 и получавших лечение - 10,5 23,9 ммоль/л, p=0,015. Более низкие значения глюкозы у лиц с ранее не диагностированным СД2 можно объяснить недавним повышением у них гликемии, с одной стороны, и недостаточно эффек-

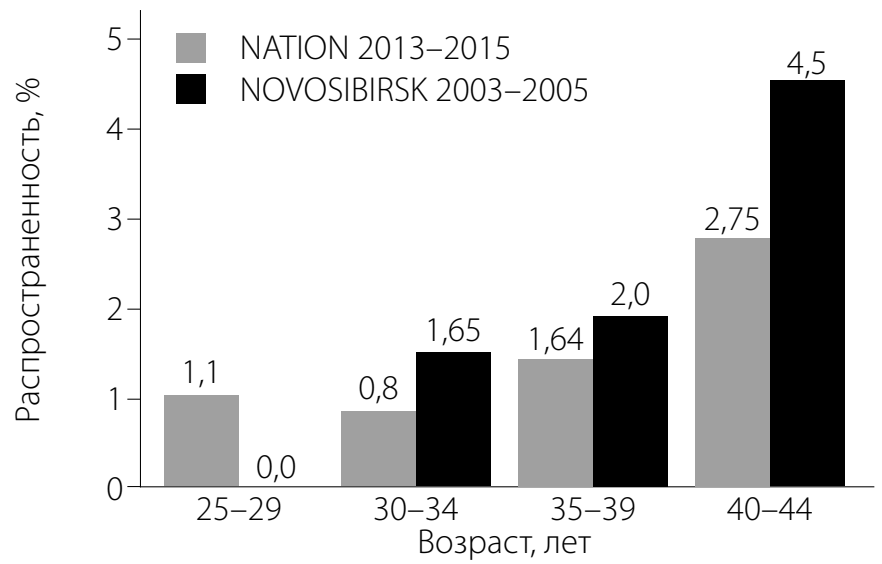

Рис. 2. Распространенность СД2 у лиц 25-44 лет. 
тивной терапией диабета у тех, кто принимал сахароснижающие препараты, - с другой [21].

Среди обследованных, знающих о наличии у них СД2, только у 18,8\% (78 человек) уровень глюкозы крови во время обследования был ниже 6,1 ммоль/л, у 30\% (124 человека) был ниже 7,0 ммоль/л. Значения глюкозы выше 211,1 ммоль/л были определены у 13,4\% (139 человек). Похожие данные приведены и в исследовании NATION, где среди участников с СД2 у 19,3\% уровень НbА был >9\%.

По сравнению с данными исследования NATION получена более высокая распространенность СД2 в возрастной группе 40-44 лет (рис. 2), что может быть объяснено разницей в подходах к диагностике СД2. В отличие от обследованных в возрасте старше 45 лет в возрастной группе 25-44 лет частота СД2 выше у мужчин, чем у женщин (за счет подгруппы 40-44 лет: 7,7 против 1,6\%). Полученные гендерные различия в частоте СД2 требуют дальнейшего анализа.

Среднепопуляционное значение уровня глюкозы натощак во всей выборке для лиц обоего пола составило 6,0 ммоль/л. Несмотря на однократное определение глюкозы крови, можно говорить о новосибирской популяции как популяции высокого риска по развитию СД (по общепринятым эпидемиологическим критериям). Данное положение подкрепляется несколькими эпидемиологическими материалами.

Во-первых, у 6,6\% населения в возрасте 45-69 лет диабет оказался выявленным впервые лишь при скрининге, что отражает частоту СД2, не диагностированного ранее.

Во-вторых, среди участников обследования в Новосибирске обнаружена высокая распространенность НГН по критерию ВО3, 1999 г. (от 6,1 до 6,9 ммоль/л), как в возрасте 45-69 лет - у 18,2\% и, что особенно вызывает тревогу, так и в молодой возрастной группе 25-44 лет - 21,6\%. В исследовании NATION у 19,3\% участников 20-79 лет был выявлен предиабет (при уровне $\mathrm{HbA}_{1 с}$ от 5,7\% до 6,5\% с ранее не диагностированным СД2).

Менее половины (4,7 из 11,3\%) обследованных в возрасте 45-69 лет и только 1 человек в возрасте 25-44 лет знали, что у них есть СД2, что свидетельствует о недостаточном уровне знаний сибиряков о проблеме СД. Такая низкая информированность о наличии СД2 свидетельствует об отсутствии настороженности, доминировании мнения у молодых жителей, что СД2 является проблемой лиц пожилого и старческого возраста. Аналогичные данные получены и в российском исследовании NATION, где процент участников в возрасте 20-79 лет с ранее не диагностированным СД2 составил 54\%.

Среди жителей Новосибирска 45-69 лет, по данным популяционного скрининга 2003-2005 гг., распространенность СД2 составила 11,3\%. Распространенность СД2 была выше в старших возрастных группах, с максимальной частотой в возрастной группе 65-69 лет - 15\%. Сопоставимость полученных данных с результатами первого национального эпидемиологического кросс-секционного исследования, проведенного на территории Российской Федерации по выявлению распространенности СД2 (NATION), позволяет оценивать полученные данные в г. Новосибирске как репрезентативные в целом для России.

\section{ЗАКЛЮЧЕНИЕ}

Распространенность СД2 по данным популяционного скрининга 2013-2015 гг. среди жителей 25-44 лет в г. Новосибирске составила 2,2\%, выше у мужчин - 3,5\%, чем у женщин - 1,1\%, $\mathrm{p} \leq 0,05$. В молодой группе обследованных с увеличением возраста мужчин росла частота СД2, максимально в возрастной группе 40-44 лет - 7,7\%. По сравнению с данными исследования NATION получена более высокая распространенность СД2 в молодых возрастных группах, что может быть объяснено разницей в подходах к диагностике СД2.

Распространенность СД2 у мужчин и женщин 45-69 лет не выявила статистически значимого отличия. В отличие от обследованных в возрасте старше 45 лет, в возрастной группе 25-44 лет частота СД2 была выше у мужчин, чем у женщин (за счет подгруппы 40-44 лет: 7,7 против 1,6\%). Полученные гендерные различия в частоте СД2 требуют дальнейшего анализа.

Эпидемиологический скрининг позволяет обнаружить в дополнение к зарегистрированным случаям такое же (по некоторымданным, в 2-3 раза большее) количество недиагностированных случаев СД. Необходимо внедрение упрощенного и удешевленного варианта эпизодических скринирующих обследований репрезентативных выборок для мониторирования эпидемиологической ситуации фактической распространенности СД, его факторов риска, прогнозирования и оценки лечебно-профилактических мероприятий.

Работу по информированности населения и скрининговое обследование нужно начинать с более молодых групп населения (с 40 лет) для ранней коррекции углеводных нарушений и проведения первичной профилактики осложнений СД.

\section{ДОПОЛНИТЕЛЬНАЯ ИНФОРМАЦИЯ}

Источник финансирования. Проект HAPIEE частично поддержан грантами Wellcome Trust, UK064947/Z/01/Z; 081081/Z/06/Z; National Institute of Aging, USA (1R01 AG23522), Российского научного фонда, № 14-45-00030. Сбор конечных точек по вновь выявленному СД2 проведен в рамках бюджетной темы регистрации № 01201282290 гос. задание № 0541-20140007.

Конфликт интересов. Авторы декларируют отсутствие явных и потенциальных конфликтов интересов, связанных с публикацией настоящей статьи.

Благодарности. Авторы выражают благодарность академику РАН Никитину Ю.П., А.М.Н., профессору Симоновой Г.И. за идею проведения данного научного исследования, д.М.Н. член-корр. РАН Рагино Ю.И. за организацию проведения биохимических исследований.

Участие авторов. С.В. Мустафина - обработка статистических данных, написание разделов материалы и методы и результатов по возрастной группе 45-69 лет, обсуждения данных; О.Д. Рымар - написание разделов введение, материалы и методы, результаты и обсуждение по возрастной группе 45-69 лет; С.К. Малютина - организатор скрининга мужчин и женщин 45-69 лет в г. Новосибирске, редактирование текста; Д.В. Денисова - организатор скрининга мужчин и женщин 25-44 лет в г Новосибирске, редактирование текста;. Л.В. Щербакова - формирование базы данных, статистический анализ, написание раздела «материалы и методы» и оформление таблиц; М.И. Воевода - идея проведения анализа данных, участие в написании «Всех разделов» и заключения. 


\section{СПИСОК ЛИТЕРАТУРЫ | REFERENCES}

1. Svo.gov.ru [интернет]. Официальный сайт полномочного представителя Президента России в Сибирском федеральном округе. [Svo.gov.ru [Internet]. Official site of the Plenipotentiary of the President of Russia in the Siberian Federal District. (In Russ.)] Доступно по: http://sfo.gov.ru. Ссылка активна на 10.05.2017.

2. Симонова Г.И., Малахина Е.С., Мустафина С.В. Сахарный диабет в практике врача-терапевта: распространенность и качество диагностики / Мониторирование сердечно-сосудистой заболеваемости, смертности и их факторов риска в разных регионах мира (проект BO3 MONICA). / Под ред. Никитина Ю.П. - Новосибирск: Гeo; 2016. - C. 579-589. [Simonova Gl, Malakhina ES, Mustafina S.V. Diabetes mellitus in the practice of a therapist: the prevalence and quality of diagnosis. In: Nikitin YP, editor. Monitoring of cardiovascular morbidity, mortality and their risk factors in different regions of the world (WHO MONICA project). Novosibirsk: Geo; 2016. p. 579-589. (In Russ.)]

3. Voevoda M., Sazonova O., Simonova G., et al. Chapter 19: Prediabetes and Diabetes Prevention Initiatives in Siberia, Russia. In: Bergman M, editor. Global Health Perspectives in Prediabetes and Diabetes Prevention. Singapore: World Scientific Publishing Co. Pte. Ltd; 2014. p. 431-447. doi: 10.1142/9789814603324_0019

4. Никитин Ю.П., Воевода М.И., Симонова Г.И. Сахарный диабет и метаболический синдром в Сибири и на Дальнем Востоке // Becmник PAMH. - 2012. - T. 67. - №1. - C. 66-74. [Nikitin YuP, Voevoda MI, Simonova GI. Diabetes mellitus and metabolic syndrome in Siberia and the Far East. Annals of the Russian Academy of Medical Sciences. 2012;67(1):66-74. (In Russ.)] doi: 10.15690/vramn.v67i1.113

5. Дедов И.И., Шестакова М.В., Викулова О.К. Государственный регистр сахарного диабета в Российской Федерации: статус 2014 г и перспективы развития // Сахарный диабет. - 2015. - Т. 18. №3. - C. 5-22. [Dedov II, Shestakova MV, Vikulova OK. National register of diabetes mellitus in Russian Federation: status on 2014. Diabetes mellitus. 2015;18(3):5-23. (In Russ.)] doi: 10.14341/DM201535-22

6. Alberti KG, Zimmet PZ. Definition, diagnosis and classification of diabetes mellitus and its complications. Part 1: diagnosis and classification of diabetes mellitus provisional report of a WHO consultation. Diabet Med. 1998;15(7):539-552. doi: 10.1002/(SICI)1096-9136(199807)15:7<539::AID-DIA668>3.0.CO;2-S

7. Genuth S, Alberti KG, Bennett P, et al. Follow-up report on the diagnosis of diabetes mellitus. Diabetes Care. 2003;26(11):3160-3167. doi: 10.2337/diacare.26.11.3160

8. American Diabetes Association. Diagnosis and Classification of Diabetes Mellitus. Diabetes Care. 2013;36(Suppl 1):67-74. doi: $10.2337 /$ dc13-S067

9. Cowie CC, Rust KF, Byrd-holt DD, et al. Prevalence of diabetes and high risk for diabetes using A1c criteria in the U.S. Population in 1988-2006. Diabetes care. 2010;33(3):562-568. doi: 10.2337/dc09-1524

10. Дедов И.И., Шестакова М.В., Галстян Г.Р. Распространенность сахарного диабета 2 типа у взрослого населения России (исследование NATION) // Cахарный диабет. - 2016. - T. 19. - №2. - C. 104-112. [Dedov II, Shestakova MV, Galstyan GR. The prevalence of type 2 diabetes mellitus in the adult population of Russia (NATION study). Diabetes mellitus. 2016;19(2):104-112. (In Russ.)] doi: 10.14341/DM2004116-17

11. Древаль А.В., Мисникова И.В., Барсуков И.А. Распространенность сахарного диабета и других нарушений углеводного обмена в зависимости от критериев диагностики // Сахарный диабет. 2010. - T. 13. - №1. - C.116-121. [Dreval AV, Misnikova IV, Barsukov IA, et al. Prevalence of diabetes mellitus and other disorders of carbohydrate metabolism depending on the diagnostic criteria. Diabetes Mellitus. 2010;13(1):116-121. (In Russ.)] doi: 10.14341/2072-0351-6026

12. Мустафина С.В, Никитин Ю.П., Симонова Г.И., Щербакова Л.В. Распространенность дислипидемий при сахарном диабете в возрасте 45-69 лет в г. Новосибирске // Атеросклероз. - 2014. - Т. 10. - №4. - C. 26-31. [Mustafina SV, Nikitin YuP, Simonova Gl, Shcherbakova LV. Prevalence of dyslipidemia in case of diabetes mellitus in Novosibirsk within patients 45-69 age. Ateroskleroz. 2014;10(4):26-31. (In Russ.)]

13. Gyberg V, De Bacquer D, De Backer G, et al. Patients with coronary arterydisease and diabetes need improved management: a report from the EUROASPIRE IV survey: a registry from the Euro Observational Research Programme of the European Society of Cardiology. Cardiovasc Diabetol. 2015;(14):133. doi: 10.1186/s12933-015-0296-y

14. Nichols M, Townsend N, Scarborough P, Rayner M. Cardiovascular disease in Europe 2014: epidemiological update. Eur Heart J. 2014;35(42):2950-2959. doi: 10.1093/eurheartj/ehu299

15. Petrukhin IS, Lunina EY. Cardiovascular disease risk factors and mortality in Russia: challenges and barriers. Public Health Rev. 2011;(33):436-449.

16. Grigoriev P, Meslé F, Shkolnikov VM, et al. The Recent Mortality Decline in Russia: Beginning of the Cardiovascular Revolution? Popul Dev Rev. 2014:40(1):107-129. doi: 10.1111/j.1728-4457.2014.00652.x

17. Peasey A., Bobak M, Kubinova R, et al. Determinants of cardiovascular disease and other non-communicable diseases in Central and Eastern Europe: Rationale and design of the HAPIEE study. BMC Public Health. 2006;(6):255. doi: 10.1186/1471-2458-6-255

18. Mozaffarian D, Benjamin EJ, Go AS, et al. Heart disease and stroke statistics--2015 update: a report from the American Heart Association. Circulation. 2015;131(4):e29-322. doi: 10.1161/CIR.0000000000000152

19. Дедов И.И., Шестакова М.В., Сунцов Ю.И., и др. Результаты реализации подпрограммы «Сахарный диабет» Федеральной целевой программы «Предупреждение и борьба с социально значимыми заболеваниями 2007-2012 годы» // Сахарный диабет. - 2013. T. 16. - №2S. - C. 1-48. [Dedov II, Shestakova MV, Suntsov YI, et al. Federal targeted programme "Prevention and Management of Socially Significant Diseases (2007-2012)": results of the "Diabetes mellitus" sub-programme. Diabetes mellitus. 2013;16(2S):1-48. (In Russ.)] doi: 10.14341/20720351-3879

20. Dedov I, Shestakova M, Benedetti MM, et al. Prevalence of type 2 diabetes mellitus (T2DM) in the adult Russian population (NATION study). Diabetes Res Clin Pract. 2016;115:90-95 doi: 10.1016/j.diabres.2016.02.010

21. Дедов И.И., Шестакова М.В., Майоров А.Ю., и др. Алгоритмы специализированной медицинской помощи больным сахарным диабетом / Под редакцией И.И. Дедова, М.В. Шестаковой, А.Ю. Майорова. - 8-й выпуск // Сахарный диабет. - 2017. - Т. 20. №1S. - C. 1-121. [Dedov II, Shestakova MV, Mayorov AY, et al. Standards of specialized diabetes care. Edited by Dedov II, Shestakova MV, Mayorov AY. 8th edition. Diabetes mellitus. 2017;20(15):1-121. (In Russ.)] doi: $10.14341 / D M 8146$

\section{ИНФОРМАЦИЯ ОБ АВТОРАХ [AUTHORS INFO]}

Мустафина Светлана Владимировна, Д.м.н. [Svetlana V. Mustafina, MD, PhD]; адрес: 630089, Новосибирск, ул. Бориса Богаткова, д. 175/1 [175/1, st. Bоrisa Bogatkova, Novosibirsk, 630089 Russian Federaiton]; ORCID: http://orcid.org/0000-0003-4716-876X; eLibrary SPIN: 8395-1395; e-mail: svetlana3548@gmail.com. Рымар Оксана Дмитриевна, д.м.н. [Oksana D. Rymar, MD, PhD]; ORCID: http://orcid.org/0000-0003-4095-0169; eLibrary SPIN: 8345-9365; e-mail: orymar23@gmail.com. Малютина Софья Константиновна, д.м.н., профессор [Sofiya K. Malyutina, MD, PhD, Professor]; eLibrary SPIN: 6780-9141; e-mail: smalyutina@hotmail.com. Денисова Диана Вахтанговна, д.м.H. [Diana V. Denisova, MD, PhD]; ORCID: http:// orcid.org/0000-0002-2470-2133; eLibrary SPIN: 9829-7692; e-mail: denisovadiana@gmail.com. Щербакова Лилия Валерьевна, с.H.c. [Liliya V. Shcherbakova, senior research associate]; ORCID: http://orcid. org/0000-0001-9270-9188; eLibrary SPIN: 5849-7040; e-mail: 9584792@mail.ru. Воевода Михаил Иванович, д.М.Н., профессор, академик PAH [Mihail I. Voevoda, MD, PhD, Professor]; ORCID: http://orcid.org/0000-0001-9425-413X; eLibrary SPIN: 6133-1780; e-mail: mvoevoda@yandex.ru.

\section{ЦИТИРОВАТЬ:}

Мустафина С.В., Рымар О.Д., Малютина С.К., Денисова Д.В., Щербакова Л.В., Воевода М.И. Распространенность сахарного диабета у взрослого населения Новосибирска // Сахарный диабет. - 2017. - T. 20. - №5. - С. 329-334. doi: 10.14341/DM8744

\section{TO CITE THIS ARTICLE:}

Mustafina SV, Rymar OD, Malyutina SK, Denisova DV, Shcherbakova LV, Voevoda MI. Prevalence of diabetes in the adult population of Novosibirsk. Diabetes mellitus. 2017;20(5):329-334. doi: 10.14341/DM874410.14341/DM9291 